\title{
Hydrocarbon Condensation in Heavy-Duty
}

\section{Diesel Exhaust}

\author{
Jyrki Ristimäki ${ }^{1 \$}$, Kati Vaaraslahti ${ }^{l+}$, Maija Lappi ${ }^{2}$, Jorma Keskinen ${ }^{l} *$ \\ ${ }^{1}$ Aerosol Physics Laboratory, Institute of Physics, Tampere University of Technology, \\ P.O. Box 692, FI-33101 Tampere, Finland \\ ${ }^{2}$ VTT Energy and Pulp\&Paper, Vehicle emissions, P.O.Box 1000, FI-02044 VTT, \\ Finland \\ ${ }^{\S}$ Current address: Wärtsilä Finland Oy, Järvikatu 2-4, P.O.Box 244, FI-65101 Vaasa, \\ Finland
}

+ Current address: Ecocat Oy, Vihtavuorentie 162, P.O.Box 20, FI-41331 Vihtavuori, Finland

*Corresponding author phone: +358-3-31152676; fax +358-3-31152600; e-mail: jorma.keskinen@tut.fi 
Table S1 Parameters describing the hydrocarbon species used in the calculation of the simulation curves.

\begin{tabular}{|l|l|l|l|}
\hline & Symbol & Value & Unit \\
\hline Density & $\rho$ & $8 \cdot 10^{2}$ & $\mathrm{~kg} / \mathrm{m}^{3}$ \\
\hline Molecule mass & M & $4 \cdot 10^{2}$ & $\mathrm{~g} / \mathrm{mol}$ \\
\hline Surface tension & $\sigma$ & $2 \cdot 10^{-2}$ & $\left(\mathrm{Ns} / \mathrm{m}^{2}\right)$ \\
\hline Diffusion coefficient & $\mathrm{D}$ & $2 \cdot 10^{-6}$ & $\mathrm{~m}^{2} / \mathrm{s}$ \\
\hline Mean free path & $\lambda$ & $4 \cdot 10^{-8}$ & $\mathrm{~m}$ \\
\hline
\end{tabular}

